TAIWANESE JOURNAL OF MATHEMATICS

Vol. 8, No. 1, pp. 103-124, March 2004

This paper is available online at http://www.math.nthu.edu.tw/tjm/

\title{
A NEW CLASS OF DOUBLY NONLINEAR EVOLUTION EQUATIONS
}

\author{
Masayasu Aso, Takesi Fukao and Nobuyuki Kenmochi
}

\begin{abstract}
In this paper we consider the solvability for a new class of doubly nonlinear evolution equations. The motivation of this work comes from a transmission problem of two degenerate parabolic equations with convection term, in which the transmission boundary is time-dependent. We give an abstract existence result, and show that the weak variational formulation for the transmission problem can be solved by applying this abstract result. In our existence proof, the abstract theory of pseudo-monotone operators is useful.
\end{abstract}

\section{INTRODUCTION}

Let $0<T<+\infty$. We consider an evolution equation of the form:

$$
\begin{gathered}
u^{\prime}(t)+K(t, \theta(t))+G(t, u(t))=f(t) \text { in } V^{*} \text { for a.e. } t \in[0, T], \\
u(0)=u_{0},
\end{gathered}
$$

where $u^{\prime}:=d u / d t$. Here, for each $t \in[0, T], K(t, \cdot)$ is a weakly continuous operator from a reflexive Banach space $V$ into its dual space $V^{*}, G(t, \cdot)$ is a weakly continuous operator from a Hilbert space $H$ into $V^{*}$, where $V$ is imbedded densely and compactly in $H, f$ is a given source function and $u_{0}$ is an initial datum. Equation (1.1) is considered with the following relation between $\theta$ and $u$ :

$$
\theta(t)=\partial \psi^{t}(u(t)) \text { in } V \text { for a.e. } t \in[0, T],
$$

where $\left\{\psi^{t}\right\}$ is a family of proper, lower semicontinuous and convex functions on space $V^{*}$ and $\partial \psi^{t}$ is the subdifferential of $\psi^{t}$ from $V^{*}$ into $V$; especially in our setting the subdifferential $\partial \psi^{t}$ is assumed to be singlevalued.

Received April 15, 2003.

Communicated by S. B. Hsu.

2000 Mathematics Subject Classification: 47J35, 35K65.

Key words and phrases: Doubly nonlinear, Pseudo-monotone, Transmission-Stefan. 
Some types of doubly nonlinear evolution equations have been considered so far, for example,

$$
A\left(u^{\prime}(t)\right)+B(u(t)) \ni f(t),
$$

which was considered by Arai [1], Senba [21], Colli and Visintin [8] and Colli [7], where $A$ and $B$ are maximal monotone, possibly nonlinear and multivalued operators from $V$ into $V^{*}$; in Colli [7], $A$ is bounded and $B$ is unbounded so that the domain $D(B)$ is contained in a Banach space $W$ imbedded compactly in $V$. Another type of doubly nonlinear evolution equations is of the form:

$$
\frac{d}{d t} A(u(t))+B(u(t)) \ni f(t),
$$

which was treated, for instance, by Kenmochi [16], Kenmochi and Pawlow [17],[18], and recently Maitre and Witomski [19]. In our problem, the unknown $u$ can be eliminated by (1.3). In fact, using the conjugate convex function $\psi^{t *}$ of $\psi^{t}$, we obtain from (1.3) that

$$
u(t) \in \partial \psi^{t *}(\theta(t)) \quad \text { in } V^{*},
$$

where $\partial \psi^{t *}$ is the subdifferential of $\psi^{t *}$ from $V$ into $V^{*}$. Hence (1.1) is formally written in the form

$$
\frac{d}{d t} \partial \psi^{t *}(\theta(t))+K(t, \theta(t))+G\left(t, \partial \psi^{t *}(\theta(t))\right) \ni f(t) \quad \text { in } V^{*}
$$

This is a new type of doubly nonlinear evolution equations in respect that the time derivative of $\partial \psi^{t *}(\theta(t))$ is included in the equation as well as a highly nonlinear perturbation $G\left(t, \partial \psi^{t *}(\theta(t))\right)$. We have not noticed any results on this class of evolution equations. In this paper we give an existence result for problem $\{(1.1)-(1.3)\}$. In our construction of a solution, we approximate (1.1) by a time discretization scheme. After getting some uniform estimates we discuss its convergences to obtain a solution of our problem.

The above type of evolution equations arises from transmission problems of two degenerate parabolic equations. This problem has been studied by Fukao, Kenmochi and Pawlow [11]. In their paper it was treated as a system of transmission-Stefan type. Two degenerate parabolic equations are combined by the transmission condition. In this case the problem is formulated as an initial value problem for (1.1) in an abstract Banach space, which will be discussed in detail in the last section.

\section{Main Result}

Throughout this paper, we denote by $V$ a real reflexive Banach space with norm $|\cdot|_{V}$, by $V^{*}$ the dual space of $V$ and by $\langle\cdot, \cdot\rangle_{V^{*}, V}$ the duality pairing between $V^{*}$ 
and $V$. Moreover, let $H$ be a real Hilbert space with inner product $(\cdot, \cdot)_{H}$ and norm $|\cdot|_{H}$ such that the following dense and compact imbeddings are satisfied:

$$
V \stackrel{d}{\hookrightarrow} H \stackrel{d}{\hookrightarrow} V^{*}
$$

Without loss of generality we may assume that $V$ and $V^{*}$ are strictly convex spaces.

Now consider the initial value problem $(\mathrm{DN}):=\{(2.1)-(2.3)\}$ :

$$
\begin{gathered}
u^{\prime}(t)+K(t, \theta(t))+G(t, u(t))=f(t) \quad \text { in } V^{*} \text { for a.e. } t \in[0, T] \\
\theta(t)=\partial \psi^{t}(u(t)) \quad \text { in } V \text { for a.e. } t \in[0, T] \\
u(0)=u_{0}
\end{gathered}
$$

under the following assumptions (A1)-(A3):

(A1) For each $t \in[0, T], \psi^{t}$ is a proper, lower semicontinuous and convex function on $V^{*}$ with $D\left(\psi^{t}\right) \subset H$, and $\psi^{t}$ is coercive, that is, there exists a positive constant $C_{0}$, independent of $t$, such that

$$
\psi^{t}(u) \geq C_{0}|u|_{H}^{2} \quad \text { for all } u \in D\left(\psi^{t}\right) .
$$

Moreover $\psi^{t}$ satisfies the following time-dependent condition: For each $t, s \in[0, T]$ and $u \in D\left(\psi^{s}\right)$, there exists $\tilde{u} \in D\left(\psi^{t}\right)$ such that

$$
\begin{gathered}
|\tilde{u}-u|_{V^{*}} \cdot C_{1}|t-s|\left(\psi^{s}(u)^{\frac{1}{2}}+1\right), \\
\psi^{t}(\tilde{u})-\psi^{s}(u) \cdot C_{1}|t-s|\left(\psi^{s}(u)+1\right),
\end{gathered}
$$

where $C_{1}$ is a positive constant independent of $t, s$ and $u$. In addition, $\psi^{t}$ is strongly monotone in the following sense:

(2.7) $\left\langle u_{1}-u_{2}, \theta_{1}-\theta_{2}\right\rangle_{V^{*}, V} \geq C_{2}\left|\theta_{1}-\theta_{2}\right|_{H}^{2} \quad$ for all $\theta_{i}=\partial \psi^{t}\left(u_{i}\right)$ and $i=1,2$.

(A2) The operator $K(t, \cdot): D(K(t, \cdot))=V \rightarrow V^{*}$ satisfies that (coerciveness)

$$
\langle K(t, \theta), \theta\rangle_{V^{*}, V} \geq C_{3}|\theta|_{V}^{2}-C_{4} \quad \text { for all } \theta \in V \text { and } t \in[0, T],
$$

(boundedness)

$$
|K(t, \theta)|_{V^{*}} \cdot C_{5}|\theta|_{V}+C_{6} \quad \text { for all } \theta \in V \text { and } t \in[0, T],
$$


where $C_{3}, C_{4}, C_{5}$ and $C_{6}$ are positive constants. Moreover $K(\cdot, \cdot)$ satisfies the following property: If $\theta_{n} \rightarrow \theta$ in $L^{2}(0, T ; H)$ and weakly in $L^{2}(0, T ; V)$ as $n \rightarrow$ $+\infty$, then

$$
K\left(\cdot, \theta_{n}(\cdot, \cdot)\right) \rightarrow K(\cdot, \theta(\cdot, \cdot)) \quad \text { weakly in } L^{2}\left(0, T ; V^{*}\right) \text { as } n \rightarrow+\infty .
$$

In addition, for each $\theta \in V, K(\cdot, \theta)$ is continuous with respect to $t$ in the following sense: There exists a continuous function $\omega:[0,+\infty) \rightarrow[0,+\infty)$ with $\omega(0)=0$ such that

$$
|K(t, \theta)-K(s, \theta)|_{V^{*}} \cdot \omega(|t-s|)\left(|\theta|_{V}+1\right) \quad \text { for all } t, s \in[0, T] .
$$

(A3) The operator $G(t, \cdot): D(G(t, \cdot))=H \rightarrow V^{*}$ satisfies that (boundedness)

$$
|G(t, u)|_{V^{*}} \cdot C_{7}|u|_{H}+C_{8} \quad \text { for all } u \in H \text { and } t \in[0, T],
$$

where $C_{7}$ and $C_{8}$ are positive constants. Moreover $G(\cdot, \cdot)$ satisfies the following property: If $t_{n} \rightarrow t$ and $u_{n} \rightarrow u$ weakly in $H$ as $n \rightarrow+\infty$, then

$$
G\left(t_{n}, u_{n}\right) \rightarrow G(t, u) \quad \text { weakly in } V^{*} \text { as } n \rightarrow+\infty \text {. }
$$

Here we give the definition of a solution of (DN).

Definition 2.1 A pair $\{u, \theta\}$ of functions $u, \theta:[0, T] \rightarrow H$ is called a solution of (DN), if $u \in W^{1,2}\left(0, T ; V^{*}\right) \cap L^{\infty}(0, T ; H), \theta \in L^{2}(0, T ; V)$ and $\{u, \theta\}$ satisfies (2.1), (2.2) and (2.3).

Our main theorem is formulated now:

Theorem 2.1 Assume that (A1)-(A3) hold. Given $f \in L^{2}\left(0, T ; V^{*}\right)$ and $u_{0} \in D\left(\psi^{0}\right)$, (DN) has at least one solution $\{u, \theta\}$.

We shall prove our existence theorem in sections 3 and 4. Our main idea for the construction of a solution is to employ the time discretization method for (DN).

\section{Approimation of (DN)}

In order to construct a solution of (DN), we use the time discretization method to approximate problem (DN). For an arbitrary $N \in \mathbf{N}$, we put $h_{N}:=T / N$, $t_{k}^{N}:=k h_{N}$ for $k=0,1, \ldots, N$ and $u_{0}^{N}:=u_{0}$. Then, we consider the following time discretization scheme for (DN): Find a pair $\left\{u_{k}^{N}, \theta_{k}^{N}\right\}$ of functions satisfying that

$$
\frac{u_{k}^{N}-u_{k-1}^{N}}{h_{N}}+K\left(t_{k}^{N}, \theta_{k}^{N}\right)=-G\left(t_{k-1}^{N}, u_{k-1}^{N}\right)+f_{k-1}^{N} \quad \text { in } V^{*}
$$


with

$$
\theta_{k}^{N}=\partial \psi^{t_{k}^{N}}\left(u_{k}^{N}\right) \quad \text { in } V \text { for } k=1,2, \ldots, N,
$$

where $f_{k}^{N}$ is the discrete approximation of $f$ given by

$$
f_{k}^{N}:=\frac{1}{h_{N}} \int_{t_{k}^{N}}^{t_{k+1}^{N}} f(s) d s \quad \text { for } k=0,1, \ldots, N-1 .
$$

Now we discuss the solvability of $(\mathrm{DN})_{N}:=\{(3.1),(3.2)\}$. For simplicity, put $A(t, \cdot):=\left(\partial \psi^{t}\right)^{-1}$. Note that $(\mathrm{DN})_{N}$ can be written in the form:

$$
A\left(t_{k}^{N}, \theta_{k}^{N}\right)+h_{N} K\left(t_{k}^{N}, \theta_{k}^{N}\right) \ni u_{k-1}^{N}-h_{N} G\left(t_{k-1}^{N}, u_{k-1}^{N}\right)+h_{N} f_{k-1}^{N} \text { in } V^{*}
$$

In order to construct the time discretization scheme, for each fixed $t_{k}^{N}$, it suffices to check the existence of a solution $\theta \in V$ of

$$
A\left(t_{k}^{N}, \theta\right)+h_{N} K\left(t_{k}^{N}, \theta\right) \ni g^{*} \text { in } V^{*},
$$

where $g^{*}$ is given in $V^{*}$. Now we recall the following general theory of nonlinear operators.

Proposition 3.1 Let $Z$ be a real reflexive Banach space and $Z^{*}$ be the dual space of $Z$. Assume that a multivalued operator $\mathcal{N}: Z \rightarrow Z^{*}$ satisfies the following conditions (N1) and (N2):

(N1) For each $z \in Z, \mathcal{N} z$ is a non-empty, bounded, convex and closed set in $V^{*}$.

(N2) $\mathcal{N}$ is weakly sequentially upper semicontinuous and it is coercive, that is,

$$
\lim _{|z|_{Z} \rightarrow+\infty} \inf _{z^{*} \in \mathcal{N} z} \frac{\left\langle z^{*}, z\right\rangle_{Z^{*}, Z}}{|z|_{Z}}=+\infty
$$

Then $\mathcal{N}$ is surjective, that is, $R(\mathcal{N})=Z^{*}$.

This proposition is well-known; for instance, it is an immediate consequence of the abstract results on the surjectiveness of nonlinear multivalued operators of Type M (see Brézis [3], Browder and Hess [6], Kenmochi [15] and so on).

Lemma 3.1 (i) Let $t \in[0, T]$ and $N \in \mathbf{N}$. Then $D(A(t, \cdot))=V$ and $A(t, \theta)+h_{N} K(t, \theta)$ is a non-empty, bounded, convex and closed set in $V^{*}$ for each $\theta \in V$.

(ii) $A(t, \cdot)+h_{N} K(t, \cdot)$ is weakly sequentially upper semicontinuous and coercive as a mapping from $V$ into $V^{*}$. 
Proof. (i) Since $A^{-1}(t, \cdot)=\partial \psi^{t}$, by (2.4) we have $R\left(A^{-1}(t, \cdot)\right)=D(A(t, \cdot))=$ $V$ and the boundedness of $A(t, \cdot)$. From the maximal monotonicity of $A(t, \cdot)$ it follows immediately that $A(t, \theta)+h_{N} K(t, \theta)$ is non-empty, bounded, convex and closed set in $V^{*}$ for all $\theta \in V$.

(ii) The coerciveness of $A(t, \cdot)+h_{N} K(t, \cdot)$ is seen from (2.8) and the monotonicity of $A(t, \cdot)$. Lastly we check that $A(t, \cdot)+h_{N} K(t, \cdot)$ is weakly sequentially upper semicontinuous in $V^{*}$. Now note that $K(t, \cdot)$ is a singlevalued and weakly continuous operator from $V$ into $V^{*}$, and $A(t, \cdot)$ is a subdifferential operator with $R(A(t, \cdot))=D\left(A^{-1}(t, \cdot)\right) \subset D\left(\psi^{t}\right) \subset H$. Therefore, by the compact imbedding $V \hookrightarrow H$, we see that the graph $G\left(A(t, \cdot)+h_{N} K(t, \cdot)\right)$ is weakly closed in $V \times V^{*}$, which implies the conclusion.

This lemma and Proposition 3.1 imply that for each fixed $N \in \mathbf{N}, k=$ $0,1, \ldots, N$, and any given $g^{*}$ in $V^{*}$, there exists $\theta \in V$ which satisfies (3.5). In our setting, the subdifferential operator $\partial \psi^{t}$ is singlevalued. Thus, Lemma 3.1 and Proposition 3.1 show that our approximation scheme $(\mathrm{DN})_{N}$ has solutions $u_{k}^{N} \in H$ and $\theta_{k}^{N} \in V$ for each $N \in \mathbf{N}$ and $k=0,1, \ldots, N$.

Moreover we obtain the following estimates for $\left\{u_{k}^{N}, \theta_{k}^{N}\right\}$.

Lemma 3.2 There exists a positive constant $M_{1}$ independent of $N$ such that

$$
\begin{aligned}
& \psi_{k}^{t_{k}^{N}}\left(u_{k}^{N}\right) \cdot M_{1} \quad \text { for all } k=0,1, \ldots, N, \\
& h_{N} \sum_{k=1}^{N}\left|\theta_{k}^{N}\right|_{V}^{2} \cdot M_{1} .
\end{aligned}
$$

Proof. Multiplying $(\mathrm{DN})_{N}$ by $\theta_{k}^{N}$ we obtain

$$
\begin{aligned}
& \frac{1}{h_{N}}\left(u_{k}^{N}, \theta_{k}^{N}\right)_{H}-\frac{1}{h_{N}}\left(u_{k-1}^{N}, \theta_{k}^{N}\right)_{H}+\left\langle K\left(t_{k}^{N}, \theta_{k}^{N}\right), \theta_{k}^{N}\right\rangle_{V^{*}, V} \\
& \quad=-\left\langle G\left(t_{k-1}^{N}, u_{k-1}^{N}\right), \theta_{k}^{N}\right\rangle_{V^{*}, V}+\left\langle f_{k-1}^{N}, \theta_{k}^{N}\right\rangle_{V^{*}, V} .
\end{aligned}
$$

By conditions (2.5) and (2.6) in (A1) for $t:=t_{k}^{N}, s:=t_{k-1}^{N}$ and $u:=u_{k-1}^{N} \in$ $D\left(\psi^{t_{k-1}^{N}}\right)$ we can find an element $\tilde{u}_{k}^{N} \in D\left(\psi^{t_{k}^{N}}\right)$ such that

$$
\begin{gathered}
\left|\tilde{u}_{k}^{N}-u_{k-1}^{N}\right|_{V^{*}} \cdot C_{1} h_{N}\left(\psi^{t_{k-1}^{N}}\left(u_{k-1}^{N}\right)^{\frac{1}{2}}+1\right), \\
\psi^{t_{k}^{N}}\left(\tilde{u}_{k}^{N}\right)-\psi^{t_{k-1}^{N}}\left(u_{k-1}^{N}\right) \cdot C_{1} h_{N}\left(\psi^{t_{k-1}^{N}}\left(u_{k-1}^{N}\right)+1\right) .
\end{gathered}
$$

By the definition of subdifferential $\partial \psi^{t_{k}^{N}}$ and (3.9) we get 


$$
\begin{aligned}
& \frac{1}{h_{N}}\left(u_{k}^{N}, \theta_{k}^{N}\right)_{H}-\frac{1}{h_{N}}\left(u_{k-1}^{N}, \theta_{k}^{N}\right)_{H} \\
& \quad=\frac{1}{h_{N}}\left(u_{k}^{N}-\tilde{u}_{k}^{N}, \theta_{k}^{N}\right)_{H}+\frac{1}{h_{N}}\left(\tilde{u}_{k}^{N}-u_{k-1}^{N}, \theta_{k}^{N}\right)_{H} \\
& \quad \geq \frac{1}{h_{N}}\left(\psi^{t_{k}^{N}}\left(u_{k}^{N}\right)-\psi^{t_{k}^{N}}\left(\tilde{u}_{k}^{N}\right)\right)-C_{1}\left(\psi^{t_{k-1}^{N}}\left(u_{k-1}^{N}\right)^{\frac{1}{2}}+1\right)\left|\theta_{k}^{N}\right|_{V} .
\end{aligned}
$$

Moreover, by Schwarz's inequality and (3.10),

$$
\begin{aligned}
\frac{1}{h_{N}}\left(\psi_{k}^{t_{k}^{N}}\left(u_{k}^{N}\right)-\psi^{t_{k}^{N}}\left(\tilde{u}_{k}^{N}\right)\right)-C_{1}\left(\psi^{t_{k-1}^{N}}\left(u_{k-1}^{N}\right)^{\frac{1}{2}}+1\right)\left|\theta_{k}^{N}\right|_{V} \\
\geq \quad \frac{1}{h_{N}}\left(\psi^{t_{k}^{N}}\left(u_{k}^{N}\right)-\psi^{t_{k-1}^{N}}\left(u_{k-1}^{N}\right)\right)+\frac{1}{h_{N}}\left(\psi^{t_{k-1}^{N}}\left(u_{k-1}^{N}\right)-\psi^{t_{k}^{N}}\left(\tilde{u}_{k}^{N}\right)\right) \\
\quad-C_{1}^{2} C_{\varepsilon_{1}} \psi^{t_{k-1}^{N}}\left(u_{k-1}^{N}\right)-\varepsilon_{1}\left|\theta_{k}^{N}\right|_{V}^{2}-C_{1}^{2} C_{\varepsilon_{1}}-\varepsilon_{1}\left|\theta_{k}^{N}\right|_{V}^{2} \\
\geq \quad \frac{1}{h_{N}}\left(\psi^{t_{k}^{N}}\left(u_{k}^{N}\right)-\psi^{t_{k-1}^{N}}\left(u_{k-1}^{N}\right)\right)-C_{1}\left(\psi^{t_{k-1}^{N}}\left(u_{k-1}^{N}\right)+1\right) \\
\quad-2 \varepsilon_{1}\left|\theta_{k}^{N}\right|_{V}^{2}-C_{1}^{2} C_{\varepsilon_{1}} \psi_{k-1}^{t_{k-1}^{N}}\left(u_{k-1}^{N}\right)-C_{1}^{2} C_{\varepsilon_{1}} \\
=\frac{1}{h_{N}}\left(\psi^{t_{k}^{N}}\left(u_{k}^{N}\right)-\psi^{t_{k-1}^{N}}\left(u_{k-1}^{N}\right)\right) \\
\quad-2 \varepsilon_{1}\left|\theta_{k}^{N}\right|_{V}^{2}-\left(C_{1}+C_{1}^{2} C_{\varepsilon_{1}}\right) \psi^{t_{k-1}^{N}}\left(u_{k-1}^{N}\right)-\left(C_{1}+C_{1}^{2} C_{\varepsilon_{1}}\right) ;
\end{aligned}
$$

hereafter $\varepsilon_{i}$ is an arbitrary positive constant and $C_{\varepsilon_{i}}:=1 /\left(4 \varepsilon_{i}\right)$ for each $i \in \mathbf{N}$. Next, by the coerciveness (2.8) of $K$ we have

$$
\left\langle K\left(t_{k}^{N}, \theta_{k}^{N}\right), \theta_{k}^{N}\right\rangle_{V^{*}, V} \geq C_{3}\left|\theta_{k}^{N}\right|_{V}^{2}-C_{4} .
$$

By the boundedness (2.12) of $G$ we have

$$
\begin{gathered}
\left\langle-G\left(t_{k-1}^{N}, u_{k-1}^{N}\right), \theta_{k}^{N}\right\rangle_{V^{*}, V}+\left\langle f_{k-1}^{N}, \theta_{k}^{N}\right\rangle_{V^{*}, V} \\
\cdot C_{7}^{2} C_{\varepsilon_{2}}\left|u_{k-1}^{N}\right|_{H}^{2}+C_{8}^{2} C_{\varepsilon_{2}}+3 \varepsilon_{2}\left|\theta_{k}^{N}\right|_{V}^{2}+C_{\varepsilon_{2}}\left|f_{k-1}^{N}\right|_{V^{*}}^{2} .
\end{gathered}
$$

We obtain from (3.8) with the help of (2.4) that

$$
\begin{aligned}
& \frac{1}{h_{N}}\left(\psi^{t_{k}^{N}}\left(u_{k}^{N}\right)-\psi^{t_{k-1}^{N}}\left(u_{k-1}^{N}\right)\right)+\left(C_{3}-2 \varepsilon_{1}-3 \varepsilon_{2}\right)\left|\theta_{k}^{N}\right|_{V}^{2} \\
& \left(C_{1}+C_{1}^{2} C_{\varepsilon_{1}}+\frac{C_{7}^{2} C_{\varepsilon_{2}}}{C_{0}}\right) \psi^{t_{k-1}^{N}}\left(u_{k-1}^{N}\right)+C_{\varepsilon_{2}}\left|f_{k-1}^{N}\right|_{V^{*}}^{2} \\
& +\left(C_{1}+C_{1}^{2} C_{\varepsilon_{1}}+C_{4}+C_{8}^{2} C_{\varepsilon_{2}}\right)
\end{aligned}
$$


where $\varepsilon_{1}$ and $\varepsilon_{2}$ are chosen so as to satisfy $C_{3}-2 \varepsilon_{1}-3 \varepsilon_{2}>0$. This inequality can be written in the form

$$
\psi^{t_{k}^{N}}\left(u_{k}^{N}\right)+M_{2} \cdot\left(1+\frac{T M_{3}}{N}\right)\left(\psi^{t_{k-1}^{N}}\left(u_{k-1}^{N}\right)+M_{2}\right) \quad \text { for all } k=1, \ldots, N,
$$

where $M_{2}$ and $M_{3}$ are positive constants independent of $N ; M_{2}$ depends on $|f|_{L^{2}\left(0, T ; V^{*}\right)}$ and $M_{3}:=C_{1}+C_{1}^{2} C_{\varepsilon_{1}}+\left(C_{7}^{2} C_{\varepsilon_{2}} / C_{0}\right)$. Here, applying the discrete Gronwall's inequality to (3.12), we obtain

$$
\psi^{t_{k}^{N}}\left(u_{k}^{N}\right) \cdot \exp \left(T M_{3}\right)\left(\psi^{0}\left(u_{0}\right)+M_{2}\right) \quad \text { for all } k=1,2, \ldots, N
$$

Next, we multiply (3.11) and (3.13) by $h_{N}$ and sum them up for $k=1,2, \ldots, N$ to obtain

$\psi^{T}\left(u_{N}^{N}\right)+\left(C_{3}-2 \varepsilon_{1}-3 \varepsilon_{2}\right) h_{N} \sum_{k=1}^{N}\left|\theta_{k}^{N}\right|_{V}^{2} \cdot M_{3} h_{N} \sum_{k=1}^{N} \psi^{t_{k-1}^{N}}\left(u_{k-1}^{N}\right)+M_{2} T+\psi^{0}\left(u_{0}\right)$.

Hence (3.7) is obtained from this inequality together with (3.13).

Now, let $\left\{u_{i}^{N}, \theta_{i}^{N}\right\}_{i=0}^{N}$ be the discretization scheme of (3.1), (3.2) and (3.3) constructed above and define the piecewise linear $H$-valued function $u^{N}$ generated by $\left\{u_{i}^{N}\right\}$ on $[0, T]$, that is, $u^{N}(0):=u_{0}$ and

$$
u^{N}(t):=\frac{\left(t-t_{k-1}^{N}\right) u_{k}^{N}+\left(t_{k}^{N}-t\right) u_{k-1}^{N}}{h_{N}} \text { if } t \in\left(t_{k-1}^{N}, t_{k}^{N}\right] \text { for } k=1,2, \ldots, N
$$

It is clear that $u^{N} \in C([0, T] ; H)$ for each $N \in \mathbf{N}$. Moreover, we have the following lemma:

Lemma 3.3 $\left\{u^{N}\right\}_{N=1}^{\infty}$ is equicontinuous as a family of functions from $[0, T]$ into $V^{*}$ and uniformly bounded on $[0, T]$ as $H$-valued functions.

Proof. Using Lemma 3.2, we have $\left|u_{k}^{N}\right|_{H} \cdot\left(M_{1} / C_{0}\right)^{1 / 2}$ for $k=1,2, \ldots, N$, so that $\left\{u^{N}\right\}$ is uniformly bounded on $[0, T]$ as $H$-valued functions. Next we take $s, t \in[0, T]$ with $s \cdot t$ such that $s \in\left[t_{k-1}^{N}, t_{k}^{N}\right]$ and $t \in\left[t_{j-1}^{N}, t_{j}^{N}\right]$, where $1 \cdot k \cdot j \cdot N$. We see from the boundedness (2.9) of $K$ and (2.12) of $G$ together with (2.4), (3.6) and (3.7) that there exists a positive constant $M_{4}$, which depends on $C_{6}$ and $C_{8}$, such that 


$$
\begin{aligned}
& \left|u^{N}(t)-u^{N}(s)\right|_{V^{*}} \\
& \quad 2 \sum_{\ell=k}^{j}\left|u_{\ell}^{N}-u_{\ell-1}^{N}\right|_{V^{*}} \\
& \text { - } 2\left(h_{N} \sum_{\ell=k}^{j}\left|K\left(t_{\ell}^{N}, \theta_{\ell}^{N}\right)\right|_{V^{*}}+h_{N} \sum_{\ell=k-1}^{j}\left|G\left(t_{\ell}^{N}, u_{\ell}^{N}\right)\right|_{V^{*}}+h_{N} \sum_{\ell=k-1}^{j}\left|f_{\ell}^{N}\right|_{V^{*}}\right) \\
& 2\left(h_{N}(j-k+1)\right)^{\frac{1}{2}}\left\{C_{5}\left(h_{N} \sum_{k=1}^{N}\left|\theta_{k}^{N}\right|_{V}^{2}\right)^{\frac{1}{2}}+C_{7}\left(h_{N} \sum_{k=1}^{N} \frac{\psi^{t_{k}^{N}}\left(u_{k}^{N}\right)}{C_{0}}\right)^{\frac{1}{2}}\right. \\
& \left.+M_{4}+|f|_{L^{2}\left(0, T ; V^{*}\right)}\right\} \\
& 2\left(|t-s|+2 h_{N}\right)^{\frac{1}{2}}\left\{C_{5} M_{1}^{\frac{1}{2}}+C_{7}\left(\frac{T M_{1}}{C_{0}}\right)^{\frac{1}{2}}+M_{4}+|f|_{L^{2}\left(0, T ; V^{*}\right)}\right\},
\end{aligned}
$$

which shows the equicontinuity of $\left\{u^{N}\right\}$ on $[0, T]$.

By virtue of Lemmas 3.2, 3.3 and the Ascoli-Arzela's theorem it is easily seen that there exists a subsequence $\left\{N_{n}\right\} \subset\{N\}$ and there exists a function $u \in$ $C\left([0, T] ; V^{*}\right)$ such that

$$
u^{N_{n}} \rightarrow u \quad \text { in } C\left([0, T] ; V^{*}\right) \text { as } n \rightarrow+\infty .
$$

Moreover, define the $V^{*}$-valued step function $\bar{u}^{N}$ and the $V$-valued step function $\bar{\theta}^{N}$ on $[0, T]$ by putting $\bar{u}^{N}(0):=u_{0}, \bar{\theta}^{N}(0):=\partial \psi^{0}\left(u_{0}\right)$ and

$$
\bar{u}^{N}(t):=u_{k}^{N}, \quad \bar{\theta}^{N}(t):=\theta_{k}^{N} \quad \text { if } t \in\left(t_{k-1}^{N}, t_{k}^{N}\right] \quad \text { for } k=1,2, \ldots, N .
$$

Then (3.14) implies

$$
\sup _{t \in[0, T]}\left|\bar{u}^{N_{n}}(t)-u(t)\right|_{V^{*}} \rightarrow 0 \quad \text { as } n \rightarrow+\infty .
$$

Next, we prove the following convergence and relation between $u$ and $\theta$ :

Lemma 3.4 There exists a subsequence $\left\{N_{m}\right\} \subset\left\{N_{n}\right\}$ with $N_{m} \rightarrow \infty$ as $m \rightarrow+\infty$ and there exists a function $\theta \in L^{2}(0, T ; V)$ such that

$$
\bar{\theta}^{N_{m}} \rightarrow \theta \quad \text { weakly in } L^{2}(0, T ; V) \text { as } m \rightarrow+\infty \text {. }
$$

Moreover the pair $\{u, \theta\}$ satisfies that $\theta(t)=\partial \psi^{t}(u(t))$ in $V$ for a.e. $t \in[0, T]$. 
Proof. By (3.7) in Lemma 3.2, we see that $\left\{\bar{\theta}^{N_{n}}\right\}_{n=1}^{\infty}=\left\{\partial \psi^{t}\left(\bar{u}^{N_{n}}\right)\right\}_{n=1}^{\infty}$ is bounded in $L^{2}(0, T ; V)$, which implies (3.17) for some subsequence $\left\{N_{m}\right\}$. Next, we show that $\theta(t)=\psi^{t}(u(t))$ for a.e. $t \in[0, T]$ which is equivalent to

$$
\begin{gathered}
\int_{0}^{T}\langle\eta(t)-u(t), \theta(t)\rangle_{V^{*}, V} d t \cdot \int_{0}^{T} \psi^{t}(\eta(t)) d t-\int_{0}^{T} \psi^{t}(u(t)) d t \\
\text { for all } \eta \in L^{2}\left(0, T ; V^{*}\right) .
\end{gathered}
$$

Let $\eta$ be any function in $L^{2}\left(0, T ; V^{*}\right)$ such that $\int_{0}^{T} \psi^{t}(\eta(t)) d t<+\infty$; hence $\eta \in L^{2}(0, T ; H)$ (cf. (2.4)). Then there are step functions $\bar{\eta}^{N_{m}} \in L^{2}(0, T ; H)$ for all $m \in \mathbf{N}$ such that

$$
\begin{gathered}
\bar{\eta}^{N_{m}} \rightarrow \eta \quad \text { in } L^{2}(0, T ; H) \text { as } m \rightarrow+\infty \\
\limsup _{m \rightarrow+\infty} \sum_{k=1}^{N_{m}} \int_{t_{k-1}^{N_{m}}}^{t_{k}^{N_{m}}} \psi^{t_{k}^{N_{m}}}\left(\bar{\eta}^{N_{m}}(t)\right) d t \cdot \int_{0}^{T} \psi^{t}(\eta(t)) d t ;
\end{gathered}
$$

see Kenmochi [16]. Now, from the definition of subdifferential it follows that

$$
\begin{gathered}
\sum_{k=1}^{N_{m}} \int_{t_{k-1}^{N_{m}}}^{t_{k}^{N_{m}}}\left\langle\bar{\eta}^{N_{m}}(t)-\bar{u}^{N_{m}}(t), \bar{\theta}^{N_{m}}(t)\right\rangle_{V^{*}, V} d t \\
\sum_{k=1}^{N_{m}} \int_{t_{k-1}^{N_{m}}}^{t_{k}^{N_{m}}} \psi^{t_{k}^{N_{m}}}\left(\bar{\eta}^{N_{m}}(t)\right) d t-\sum_{k=1}^{N_{m}} \int_{t_{k-1}^{N_{m}}}^{t_{k}^{N_{m}}} \psi^{t_{k}^{N_{m}}}\left(\bar{u}^{N_{m}}(t)\right) d t .
\end{gathered}
$$

Let $t$ be any time in $(0, T]$. By virtue of (2.5) and (2.6) in (A1) for $t \in\left(t_{k-1}^{N_{m}}, t_{k}^{N_{m}}\right]$, $s:=t_{k}^{N_{m}}$ and $\bar{u}^{N_{m}}(t) \in D\left(\psi^{t_{k}^{N_{m}}}\right)$ there exists a function $\tilde{z}_{m} \in D\left(\psi^{t}\right)$ such that

$$
\begin{gathered}
\left|\tilde{z}_{m}-\bar{u}^{N_{m}}\left(t_{k}^{N_{m}}\right)\right|_{V^{*}} \cdot C_{1}\left|t-t_{k}^{N_{m}}\right|\left(\psi^{t_{k}^{N_{m}}}\left(\bar{u}^{N_{m}}\left(t_{k}^{N_{m}}\right)\right)^{\frac{1}{2}}+1\right), \\
\psi^{t}\left(\tilde{z}_{m}\right)-\psi^{t_{k}^{N_{m}}}\left(\bar{u}^{N_{m}}\left(t_{k}^{N_{m}}\right)\right) \cdot C_{1}\left|t-t_{k}^{N_{m}}\right|\left(\psi^{t_{k}^{N_{m}}}\left(\bar{u}^{N_{m}}\left(t_{k}^{N_{m}}\right)\right)+1\right) .
\end{gathered}
$$

We now observe from (3.16) and (3.22) that

$$
\tilde{z}_{m} \rightarrow u(t) \text { in } V^{*} \text { as } m \rightarrow+\infty .
$$

On the other hand, using (3.23), (3.24) and the fact that $\psi^{t}$ is lower semicontinuous on $V^{*}$, we see

$$
\begin{aligned}
& \liminf _{m \rightarrow+\infty} \psi_{k}^{t_{k}^{N m}}\left(\bar{u}^{N_{m}}\left(t_{k}^{N_{m}}\right)\right) \\
& \quad \geq \liminf _{m \rightarrow+\infty}\left(\frac{1}{1+C_{1}\left|t-t_{k}^{N_{m}}\right|} \psi^{t}\left(\tilde{z}_{m}\right)-\frac{C_{1}\left|t-t_{k}^{N_{m}}\right|}{1+C_{1}\left|t-t_{k}^{N_{m}}\right|}\right) \\
& \geq \psi^{t}(u(t)) .
\end{aligned}
$$


Thus, taking the limit as $m \rightarrow+\infty$ in (3.21) and using (3.16), (3.17), (3.19) and (3.20) with the help of Fatou's lemma, we get (3.18).

\section{Proof of THEOREM}

In this section we prove the main theorem after preparing two lemmas. Let $\left\{\bar{u}^{N_{m}}, \bar{\theta}^{N_{m}}\right\}_{m=1}^{\infty}$ and $\{u, \theta\}$ be such as obtained in the last section.

Lemma 4.1 Let $\left\{\theta_{j}\right\}$ be any sequence in $C([0, T] ; V)$ such that $\theta_{j} \rightarrow \theta$ in $L^{2}(0, T ; V)$ as $j \rightarrow+\infty$. Put

$$
u_{j}(t):=\left(\partial \psi^{t}+F^{-1}\right)^{-1}\left(\theta_{j}(t)+F^{-1} u(t)\right) \quad \text { in } V^{*} \text { for all } t \in[0, T] .
$$

where $F$ is the duality mapping from $V$ to $V^{*}$. Then $u_{j} \in C\left([0, T] ; V^{*}\right)$ and

$$
u_{j} \rightarrow u \text { weakly in } L^{2}\left(0, T ; V^{*}\right) \text { as } j \rightarrow+\infty \text {. }
$$

Proof. First note that $\partial \psi^{t}+F^{-1}$ is a singlevalued and surjective operator from $V^{*}$ into $V$ as well as from $L^{2}\left(0, T ; V^{*}\right)$ into $L^{2}(0, T ; V)$. Clearly $u_{j} \in$ $L^{2}\left(0, T ; V^{*}\right)$. We show $u_{j} \in C\left([0, T] ; V^{*}\right)$ as follows: For each fixed $j \in \mathbf{N}$ and $t \in[0, T]$, let $\left\{t_{n}\right\} \subset[0, T]$ be any sequence such that $t_{n} \rightarrow t$ as $n \rightarrow+\infty$. Our claim is to show that

$$
u_{j}\left(t_{n}\right) \rightarrow u_{j}(t) \quad \text { in } V^{*} \text { as } n \rightarrow+\infty .
$$

Take a function $w \in C\left([0, T] ; V^{*}\right)$ with $w(t) \in D\left(\psi^{t}\right)$ such that

$$
t \rightarrow \psi^{t}(w(t)) \text { is bounded in }[0, T],
$$

for example, by a result in Kenmochi [16] we can find $w$ as a solution of the Cauchy problem

$$
\begin{gathered}
\frac{\partial w}{\partial t}(t)+\partial_{H} \psi^{t}(w(t))=0 \text { in } H, \\
w(0)=w_{0} \quad \text { in } H,
\end{gathered}
$$

where $\partial_{H} \psi^{t}$ is the subdifferential of $\psi^{t}$ in $H$ and $w_{0}$ is an element of $D\left(\psi^{0}\right)$. Put $g_{j}(t):=\theta_{j}(t)+F^{-1} u(t)$ in $V$ for all $t \in[0, T]$. Then, from the definition of subdifferential and assumption (2.4) in (A1) it follows that

$$
\begin{aligned}
& \left\langle u_{j}\left(t_{n}\right)-w\left(t_{n}\right), g_{j}\left(t_{n}\right)\right\rangle_{V^{*}, V} \\
& \quad=\left\langle u_{j}\left(t_{n}\right)-w\left(t_{n}\right), \partial \psi^{t_{n}}\left(u_{j}\left(t_{n}\right)\right)+F^{-1} u_{j}\left(t_{n}\right)\right\rangle_{V^{*}, V} \\
& \quad \geq \psi^{t_{n}}\left(u_{j}\left(t_{n}\right)\right)-\psi^{t_{n}}\left(w\left(t_{n}\right)\right)+\left|u_{j}\left(t_{n}\right)\right|_{V^{*}}^{2}-\left|w\left(t_{n}\right)\right|_{V^{*}}\left|u_{j}\left(t_{n}\right)\right|_{V^{*}} \\
& \quad \geq C_{0}\left|u_{j}\left(t_{n}\right)\right|_{H}^{2}-\psi^{t_{n}}\left(w\left(t_{n}\right)\right)+\left|u_{j}\left(t_{n}\right)\right|_{V^{*}}^{2}-\frac{1}{2}\left|w\left(t_{n}\right)\right|_{V^{*}}^{2}-\frac{1}{2}\left|u_{j}\left(t_{n}\right)\right|_{V^{*}}^{2} \\
& \quad \geq C_{0}\left|u_{j}\left(t_{n}\right)\right|_{H}^{2}-\psi^{t_{n}}\left(w\left(t_{n}\right)\right)-\frac{1}{2}\left|w\left(t_{n}\right)\right|_{V^{*}}^{2} .
\end{aligned}
$$


On the other hand, there exists a positive constant $M_{5}$ such that

$$
\begin{array}{ll}
\left\langle u_{j}\left(t_{n}\right)-w\left(t_{n}\right), g_{j}\left(t_{n}\right)\right\rangle_{V^{*}, V} \\
\quad & M_{5}\left|u_{j}\left(t_{n}\right)\right|_{H}\left|g_{j}\left(t_{n}\right)\right|_{V}+\left|w\left(t_{n}\right)\right|_{V^{*}}\left|g_{j}\left(t_{n}\right)\right|_{V} \\
. & M_{5}^{2} \varepsilon_{3}\left|u_{j}\left(t_{n}\right)\right|_{H}^{2}+C_{\varepsilon_{3}}\left|g_{j}\left(t_{n}\right)\right|_{V}^{2}+\frac{1}{2}\left|w\left(t_{n}\right)\right|_{V^{*}}^{2}+\frac{1}{2}\left|g_{j}\left(t_{n}\right)\right|_{V}^{2} .
\end{array}
$$

Since $\left|g_{j}(\tau)\right|_{V} \cdot\left|\theta_{j}(\tau)\right|_{V}+|u(\tau)|_{V^{*}}=: M_{6}$ for all $\tau \in[0, T]$, we have for any $\varepsilon_{3}>0$ that

$$
\begin{aligned}
\left(C_{0}-M_{5}^{2} \varepsilon_{3}\right)\left|u_{j}\left(t_{n}\right)\right|_{H}^{2} & \cdot \quad \psi^{t_{n}}\left(w\left(t_{n}\right)\right)+\left|w\left(t_{n}\right)\right|_{V^{*}}^{2}+\left(C_{\varepsilon_{3}}+\frac{1}{2}\right)\left|g_{j}\left(t_{n}\right)\right|_{V}^{2} \\
& \cdot \max _{\tau \in[0, T]}\left\{\psi^{\tau}(w(\tau))+|w(\tau)|_{V^{*}}^{2}\right\}+\left(C_{\varepsilon_{3}}+\frac{1}{2}\right) M_{6}^{2} .
\end{aligned}
$$

Therefore $\left\{u_{j}\left(t_{n}\right)\right\}_{n=1}^{\infty}$ is bounded in $H$. Hence, the compact imbedding $H \hookrightarrow V^{*}$ implies that there exist a subsequence $\left\{t_{n_{i}}\right\} \subset\left\{t_{n}\right\}$ with $t_{n_{i}} \rightarrow t$ as $i \rightarrow+\infty$ and an element $z_{j}(t) \in V^{*}$ such that

$$
u_{j}\left(t_{n_{i}}\right) \rightarrow z_{j}(t) \quad \text { in } V^{*} \text { as } i \rightarrow+\infty .
$$

Now, let $i \rightarrow+\infty$ in the relation

$$
\partial \psi^{t_{n_{i}}}\left(u_{j}\left(t_{n_{i}}\right)\right)+F^{-1} u_{j}\left(t_{n_{i}}\right)=g_{j}\left(t_{n_{i}}\right) \quad \text { in } V \text { for all } i \in \mathbf{N} .
$$

Then, (4.4) and (4.5) imply that

$$
\partial \psi^{t}\left(z_{j}(t)\right)+F^{-1} z_{j}(t)=g_{j}(t) \quad \text { in } V .
$$

On the other hand, we have by (4.1) that

$$
\begin{aligned}
g_{j}(t) & :=\theta_{j}(t)+F^{-1} u(t) \\
& =\left\{\partial \psi^{t}\left(u_{j}(t)\right)+F^{-1} u_{j}(t)-F^{-1} u(t)\right\}+F^{-1} u(t) \\
& =\partial \psi^{t}\left(u_{j}(t)\right)+F^{-1} u_{j}(t) .
\end{aligned}
$$

On account of the strict monotonicity of $\partial \psi^{t}+F^{-1},(4.6)$ implies that $z_{j}(t)=u_{j}(t)$. Therefore we see that $u_{j}$ is continuous in $V^{*}$ at $t$. Thus $u_{j} \in C\left([0, T] ; V^{*}\right)$. Finally letting $j \rightarrow+\infty$ in (4.1), we obtain (4.2).

Lemma 4.2 There exists a subsequence $\left\{N_{m}\right\} \subset\left\{N_{n}\right\}$ with $N_{m} \rightarrow+\infty$ as $m \rightarrow+\infty$ such that

$$
\bar{\theta}^{N_{m}} \rightarrow \theta \quad \text { in } L^{2}(0, T ; H) \text { as } m \rightarrow+\infty .
$$


Proof. Using Lemma 4.1, we consider step functions $\bar{u}_{j}^{N_{m}}$ and $\bar{\theta}_{j}^{N_{m}}$ given by

$$
\begin{aligned}
\bar{u}_{j}^{N_{m}}(t):= & u_{j}\left(t_{k}^{N_{m}}\right) \quad \text { if } t \in\left(t_{k-1}^{N_{m}}, t_{k}^{N_{m}}\right] \quad \text { for } k=1,2, \ldots, N_{m}, \\
\bar{\theta}_{j}^{N_{m}}(t):= & \theta_{j}\left(t_{k}^{N_{m}}\right) \\
= & \partial \psi^{t_{k}^{N_{m}}}\left(u_{j}\left(t_{k}^{N_{m}}\right)\right)+F^{-1} u_{j}\left(t_{k}^{N_{m}}\right)-F^{-1} u\left(t_{k}^{N_{m}}\right) \\
& \quad \text { if } t \in\left(t_{k-1}^{N_{m}}, t_{k}^{N_{m}}\right] \quad \text { for } k=1,2, \ldots, N_{m} .
\end{aligned}
$$

Then, for each $j \in \mathbf{N}$ we see from the above definitions that

$$
\begin{gathered}
\bar{u}_{j}^{N_{m}}(t) \rightarrow u_{j}(t) \quad \text { in } V^{*} \text { uniformly } t \in[0, T], \\
\bar{\theta}_{j}^{N_{m}}(t) \rightarrow \theta_{j}(t) \quad \text { in } V \text { uniformly } t \in[0, T],
\end{gathered}
$$

as $m \rightarrow+\infty$. Now we note that

$$
\begin{aligned}
I:= & \int_{0}^{T}\left\langle\bar{u}^{N_{m}}(t)-u(t), \bar{\theta}^{N_{m}}(t)-\theta(t)\right\rangle_{V^{*}, V} d t \\
= & \int_{0}^{T}\left\langle\bar{u}^{N_{m}}(t)-\bar{u}_{j}^{N_{m}}(t), \bar{\theta}^{N_{m}}(t)-\theta(t)\right\rangle_{V^{*}, V} d t \\
& +\int_{0}^{T}\left\langle\bar{u}_{j}^{N_{m}}(t)-u_{j}(t), \bar{\theta}^{N_{m}}(t)-\theta(t)\right\rangle_{V^{*}, V} d t \\
& \quad+\int_{0}^{T}\left\langle u_{j}(t)-u(t), \bar{\theta}^{N_{m}}(t)-\theta(t)\right\rangle_{V^{*}, V} d t \\
=: & I_{1}+I_{2}+I_{3} .
\end{aligned}
$$

The first term $I_{1}$ of $I$ is estimated as follows: With the help of the strong monotonicity (2.7) in (A1) we have

$$
\begin{aligned}
I_{1}= & \int_{0}^{T}\left\langle\bar{u}^{N_{m}}(t)-\bar{u}_{j}^{N_{m}}(t), \bar{\theta}^{N_{m}}(t)-\theta(t)\right\rangle_{V^{*}, V} d t \\
= & h_{N_{m}} \sum_{k=1}^{N_{m}}\left\langle\bar{u}^{N_{m}}\left(t_{k}^{N_{m}}\right)-\bar{u}_{j}^{N_{m}}\left(t_{k}^{N_{m}}\right), \bar{\theta}^{N_{m}}\left(t_{k}^{N_{m}}\right)-\bar{\theta}_{j}^{N_{m}}\left(t_{k}^{N_{m}}\right)\right\rangle_{V^{*}, V} \\
& +\int_{0}^{T}\left\langle\bar{u}^{N_{m}}(t)-\bar{u}_{j}^{N_{m}}(t), \bar{\theta}_{j}^{N_{m}}(t)-\theta_{j}(t)\right\rangle_{V^{*}, V} d t \\
& +\int_{0}^{T}\left\langle\bar{u}^{N_{m}}(t)-\bar{u}_{j}^{N_{m}}(t), \theta_{j}(t)-\theta(t)\right\rangle_{V^{*}, V} d t
\end{aligned}
$$




$$
\begin{aligned}
\geq & C_{2} \int_{0}^{T}\left|\bar{\theta}^{N_{m}}(t)-\bar{\theta}_{j}^{N_{m}}(t)\right|_{H}^{2} d t \\
& +C_{2} \int_{0}^{T}\left\langle\bar{u}^{N_{m}}(t)-\bar{u}_{j}^{N_{m}}(t), F^{-1} u(t)-F^{-1} \bar{u}_{j}^{N_{m}}(t)\right\rangle_{V^{*}, V} d t \\
& +\int_{0}^{T}\left\langle\bar{u}^{N_{m}}(t)-\bar{u}_{j}^{N_{m}}(t), \bar{\theta}_{j}^{N_{m}}(t)-\theta_{j}(t)\right\rangle_{V^{*}, V} d t \\
& +\int_{0}^{T}\left\langle\bar{u}^{N_{m}}(t)-\bar{u}_{j}^{N_{m}}(t), \theta_{j}(t)-\theta(t)\right\rangle_{V^{*}, V} d t \\
\geq & \frac{C_{2}}{3} \int_{0}^{T}\left|\bar{\theta}^{N_{m}}(t)-\theta(t)\right|_{H}^{2} d t \\
& -C_{2} \int_{0}^{T}\left|\bar{\theta}_{j}^{N_{m}}(t)-\theta_{j}(t)\right|_{H}^{2} d t-C_{2} \int_{0}^{T}\left|\theta_{j}(t)-\theta(t)\right|_{H}^{2} d t \\
& +C_{2} \int_{0}^{T}\left\langle\bar{u}^{N_{m}}(t)-\bar{u}_{j}^{N_{m}}(t), F^{-1} u(t)-F^{-1} \bar{u}_{j}^{N_{m}}(t)\right\rangle_{V^{*}, V} d t \\
& +\int_{0}^{T}\left\langle\bar{u}^{N_{m}}(t)-\bar{u}_{j}^{N_{m}}(t), \bar{\theta}_{j}^{N_{m}}(t)-\theta_{j}(t)\right\rangle_{V^{*}, V} d t \\
& +\int_{0}^{T}\left\langle\bar{u}^{N_{m}}(t)-\bar{u}_{j}^{N_{m}}(t), \theta_{j}(t)-\theta(t)\right\rangle_{V^{*}, V} d t .
\end{aligned}
$$

Adding the second and third terms $I_{2}$ and $I_{3}$ to the above inequality, we obtain

$$
\begin{aligned}
& \frac{C_{2}}{3} \int_{0}^{T}\left|\bar{\theta}^{N_{m}}(t)-\theta(t)\right|_{H}^{2} d t \\
& \cdot \quad I-I_{2}-I_{3}+C_{2} \int_{0}^{T}\left|\theta(t)-\theta_{j}(t)\right|_{H}^{2} d t+C_{2} \int_{0}^{T}\left|\theta_{j}(t)-\bar{\theta}_{j}^{N_{m}}(t)\right|_{H}^{2} d t \\
& \quad-C_{2} \int_{0}^{T}\left\langle\bar{u}^{N_{m}}(t)-\bar{u}_{j}^{N_{m}}(t), F^{-1} u(t)-F^{-1} \bar{u}_{j}^{N_{m}}(t)\right\rangle_{V^{*}, V} d t \\
& \quad-\int_{0}^{T}\left\langle\bar{u}^{N_{m}}(t)-\bar{u}_{j}^{N_{m}}(t), \bar{\theta}_{j}^{N_{m}}(t)-\theta_{j}(t)\right\rangle_{V^{*}, V} d t \\
& \quad-\int_{0}^{T}\left\langle\bar{u}^{N_{m}}(t)-\bar{u}_{j}^{N_{m}}(t), \theta_{j}(t)-\theta(t)\right\rangle_{V^{*}, V} d t .
\end{aligned}
$$

Now, let $m \rightarrow+\infty$. Then, by convergences (3.16), (3.17), (4.8), (4.9) and the monotonicity of $F^{-1}$, 


$$
\begin{aligned}
\limsup _{m \rightarrow+\infty} & \int_{0}^{T}\left|\bar{\theta}^{N_{m}}(t)-\theta(t)\right|_{H}^{2} d t \\
\cdot \quad & 3 \int_{0}^{T}\left|\theta(t)-\theta_{j}(t)\right|_{H}^{2} d t \\
& -3 \int_{0}^{T}\left\langle u(t)-u_{j}(t), F^{-1} u(t)-F^{-1} u_{j}(t)\right\rangle_{V^{*}, V} d t \\
& -\frac{3}{C_{2}} \int_{0}^{T}\left\langle u(t)-u_{j}(t), \theta_{j}(t)-\theta(t)\right\rangle_{V^{*}, V} d t \\
& 3 \int_{0}^{T}\left|\theta(t)-\theta_{j}(t)\right|_{H}^{2} d t \\
& -\frac{3}{C_{2}} \int_{0}^{T}\left\langle u(t)-u_{j}(t), \theta_{j}(t)-\theta(t)\right\rangle_{V^{*}, V} d t \quad \text { for all } j \in \mathbf{N} .
\end{aligned}
$$

Moreover, let $j \rightarrow+\infty$ in the above inequality. Then, by virtue of Lemma 4.1, we obtain that

$$
\limsup _{m \rightarrow+\infty} \int_{0}^{T}\left|\bar{\theta}^{N_{m}}(t)-\theta(t)\right|_{H}^{2} d t \cdot 0 .
$$

Thus $\bar{\theta}^{N_{m}} \rightarrow \theta$ in $L^{2}(0, T ; H)$ as $m \rightarrow+\infty$.

Proof of Theorem 2.1 As was shown above, there exist a subsequence $\left\{N_{m}\right\} \subset\{N\}, \theta \in L^{2}(0, T ; V)$ and $u \in C\left([0, T] ; V^{*}\right) \cap L^{\infty}(0, T ; H)$ such that

$$
\begin{gathered}
\bar{\theta}^{N_{m}} \rightarrow \theta \quad \text { in } L^{2}(0, T ; H), \\
\bar{\theta}^{N_{m}} \rightarrow \theta \quad \text { weakly in } L^{2}(0, T ; V), \\
u^{N_{m}} \rightarrow u \quad \text { in } C\left([0, T] ; V^{*}\right) \quad \text { as } m \rightarrow+\infty,
\end{gathered}
$$

and

$$
\theta(t)=\partial \psi^{t}(u(t)) \quad \text { in } V \text { for a.e. } t \in[0, T] .
$$

Now, define $V^{*}$-valued step functions $\bar{K}^{N_{m}}, \bar{G}^{N_{m}}$ and $\bar{f}^{N_{m}}$ on $[0, T]$ by

$$
\begin{gathered}
\bar{K}^{N_{m}}(t)=K\left(t_{k}^{N_{m}}, \theta_{k}^{N_{m}}\right) \quad \text { if } t \in\left(t_{k-1}^{N_{m}}, t_{k}^{N_{m}}\right] \quad \text { for } k=1,2, \ldots, N_{m}, \\
\bar{G}^{N_{m}}(t)=G\left(t_{k}^{N_{m}}, u_{k}^{N_{m}}\right) \quad \text { if } t \in\left(t_{k-1}^{N_{m}}, t_{k}^{N_{m}}\right] \quad \text { for } k=1,2, \ldots, N_{m}, \\
\bar{f}^{N_{m}}(t)=f_{k}^{N_{m}} \quad \text { if } t \in\left(t_{k-1}^{N_{m}}, t_{k}^{N_{m}}\right] \quad \text { for } k=1,2, \ldots, N_{m} .
\end{gathered}
$$


Then our approximate problem $(\mathrm{DN})_{N_{m}}$ can be written in the form:

$$
\frac{d}{d t} u^{N_{m}}(t)+\bar{K}^{N_{m}}(t)+\bar{G}^{N_{m}}(t)=\bar{f}^{N_{m}}(t) \quad \text { in } V^{*} \text { for a.e. } t \in[0, T] .
$$

Our claim is to show that (4.10) converges to (2.1) as $m \rightarrow+\infty$. To this end, we show that

$$
\bar{K}^{N_{m}} \rightarrow K(\cdot, \theta(\cdot)) \quad \text { weakly in } L^{2}\left(0, T ; V^{*}\right) \text { as } m \rightarrow+\infty .
$$

By (2.10) in the assumptions (A2) of $K$ we see that

$$
K\left(\cdot, \bar{\theta}^{N_{m}}(\cdot)\right) \rightarrow K(\cdot, \theta(\cdot)) \quad \text { weakly in } L^{2}\left(0, T ; V^{*}\right) \text { as } m \rightarrow+\infty .
$$

Moreover, by (2.11) in the assumptions (A2) of $K$,

$$
\begin{gathered}
\left|\bar{K}^{N_{m}}(t)-K\left(t, \bar{\theta}^{N_{m}}(t)\right)\right|_{V^{*}} \cdot \omega\left(\left|t_{k}^{N_{m}}-t\right|\right)\left(\left|\bar{\theta}^{N_{m}}(t)\right|_{V}+1\right) \\
\text { for all } t \in\left(t_{k-1}^{N_{m}}, t_{k}^{N_{m}}\right] \text { and } k=1,2, \ldots, N_{m} .
\end{gathered}
$$

Hence (4.11) holds. Similarly, by (2.13) in the assumption (A3) of $G$, we have

$$
\bar{G}^{N_{m}} \rightarrow G(\cdot, u(\cdot)) \quad \text { weakly in } L^{2}\left(0, T ; V^{*}\right) \text { as } m \rightarrow+\infty,
$$

and clearly

$$
\bar{f}^{N_{m}} \rightarrow f \quad \text { in } L^{2}\left(0, T ; V^{*}\right) \text { as } m \rightarrow+\infty .
$$

These convergences imply that $\left\{(d / d t) u^{N_{m}}\right\}$ is bounded in $L^{2}\left(0, T ; V^{*}\right)$, so that

$$
\frac{d}{d t} u^{N_{m}} \rightarrow u^{\prime} \quad \text { weakly in } L^{2}\left(0, T ; V^{*}\right) \text { as } m \rightarrow+\infty,
$$

Finally, passing to the limit in $m$ of (4.10), we have

$$
u^{\prime}(t)+K(t, \theta(t))+G(t, u(t))=f(t) \quad \text { in } V^{*} \text { for a.e. } t \in[0, T] .
$$

Thus $\{u, \theta\}$ is a solution of (DN).

\section{APPLICATION}

In this section we give an application of our abstract result.

Let $0<T<+\infty$, and $\subset \mathbf{R}^{3}$ be a bounded domain with smooth boundary $\Gamma:=\partial$. Assume that for each $t \in[0, T], \quad$ is divided into two subdomains ${ }_{1}(t)$ and ${ }_{2}(t)$ by a time-dependent interface $\Gamma_{12}(t)$, that is,

$$
={ }_{1}(t) \cup \Gamma_{12}(t) \cup{ }_{2}(t) \text { for all } t \in[0, T] .
$$

We denote by $\Gamma_{i}(t)$ the set $\partial{ }_{i}(t) \backslash \Gamma_{12}(t)$ for $i=1,2$ (see Fig. 1). 


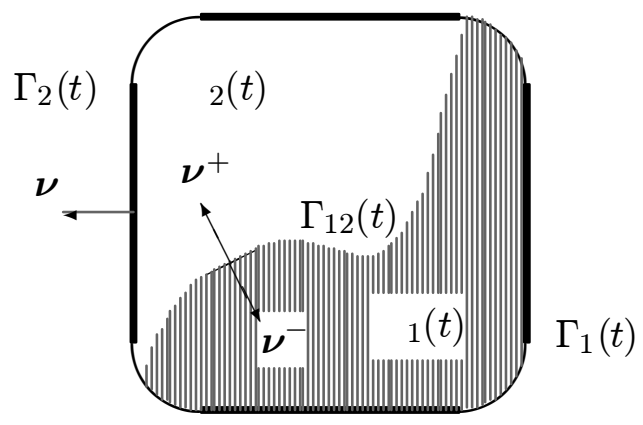

Fig. 1

Under this setting of the domains, we consider the following system of two degenerate parabolic equations:

(5.1) $\left(\frac{\partial u_{1}}{\partial t}+\mathbf{v}_{1} \cdot \nabla u_{1}\right)-\operatorname{div}\left(k_{1}\left(\theta_{1}\right) \nabla \theta_{1}\right)=h \quad$ in $Q_{1}:=\bigcup_{t \in(0, T)}\{t\} \times{ }_{1}(t)$,

$$
\theta_{1}=\beta_{1}\left(u_{1}\right) \quad \text { in } Q_{1}
$$

$$
\begin{gathered}
\left(\frac{\partial u_{2}}{\partial t}+\mathbf{v}_{2} \cdot \nabla u_{2}\right)-\operatorname{div}\left(k_{2}\left(\theta_{2}\right) \nabla \theta_{2}\right)=h \quad \text { in } Q_{2}:=\bigcup_{t \in(0, T)}\{t\} \times{ }_{2}(t) \\
\theta_{2}=\beta_{2}\left(u_{2}\right) \text { in } Q_{2}
\end{gathered}
$$

where div means the divergence with respect to space variable $x, h$ is a given function in $Q:=(0, T) \times$, and for $i=1,2, \mathbf{v}_{i}$ are given vector fields on $\bar{Q}_{i}$. Moreover, for each $i=1,2, \beta_{i}$ and $k_{i}$ are functions such that

- $\beta_{i}: \mathbf{R} \rightarrow \mathbf{R}$ is Lipschitz continuous and non-decreasing, and satisfies that

$$
\left|\beta_{i}(r)\right| \geq C_{\beta_{i}}|r|-C_{\beta_{i}}^{\prime} \quad \text { for all } r \in \mathbf{R},
$$

where $C_{\beta_{i}}$ and $C_{\beta_{i}}^{\prime}$ are positive constants;

- $k_{i}: \mathbf{R} \rightarrow \mathbf{R}$ is positive and non-decreasing, and satisfies that

$$
0<C_{k_{i}} \cdot k_{i}(r) \cdot C_{k_{i}}^{\prime} \quad \text { for all } r \in \mathbf{R},
$$

where $C_{k_{i}}$ and $C_{k_{i}}^{\prime}$ are positive constants. 
We combine these two equations (5.1) and (5.3) on the moving boundary $\Gamma_{12}(t)$ by the transmission condition

$$
\theta_{1}=\theta_{2}, \quad k_{1}\left(\theta_{1}\right) \frac{\partial \theta_{1}}{\partial \boldsymbol{\nu}^{+}}+k_{2}\left(\theta_{2}\right) \frac{\partial \theta_{2}}{\partial \boldsymbol{\nu}^{-}}=0 \quad \text { on } \Sigma_{12}:=\bigcup_{t \in(0, T)}\{t\} \times \Gamma_{12}(t)
$$

where $\boldsymbol{\nu}^{+}:=\boldsymbol{\nu}^{+}(t, x)$ is the unit normal vector on $\Gamma_{12}(t)$ pointing to ${ }_{2}(t)$ and $\nu^{-}:=-\nu^{+}$. We consider the system $\{(5.1)-(5.4),(5.7)\}$, subject to the initial and boundary conditions for $i=1,2$ :

$$
\begin{gathered}
u_{i}(0)=u_{i 0} \quad \text { in } \quad{ }_{i}(0), \\
k_{i}\left(\theta_{i}\right) \frac{\partial \theta_{i}}{\partial \boldsymbol{\nu}}+n_{0} \theta_{i}=p \quad \text { on } \Sigma_{i}:=\bigcup_{t \in(0, T)}\{t\} \times \Gamma_{i}(t),
\end{gathered}
$$

where $u_{0}$ and $p$ are given functions, $n_{0}$ is a positive constant and $\boldsymbol{\nu}:=\boldsymbol{\nu}(x)$ is the unit vector outward normal to $\Gamma$. The system $\{(5.1)-(5.4),(5.7)-(5.9)\}$ is referred as (TDP).

It is well-known that equations (5.1) and (5.2) (resp. (5.3) and (5.4)) describe a Stefan problem in non-cylindrical domain $Q_{1}$ (resp. $Q_{2}$ ), in which there is a convective vector field $\mathbf{v}_{1}$ (resp. $\mathbf{v}_{2}$ ). We call such a problem the transmissionStefan problem. This problem is discussed under the following assumptions (A4) and (A5) (cf. [10,11]):

(A4) The domain ${ }_{1}(t)$ and ${ }_{2}(t)$ depend smoothly on time $t$ in the following sense: There is a transformation $y=\mathbf{y}(t, x):=\left(y_{1}(t, x), y_{2}(t, x), y_{3}(t, x)\right)$ which is a function of $C^{2}$-class from $\bar{Q}$ into $\mathbf{R}^{3}$ satisfying that $y=\mathbf{y}(t, x)$ is a $C^{2}$ diffeomorphism from ${ }^{-}$onto itself for each $t \in[0, T]$ and

$\mathbf{y}(t, \overline{i(t)})=\overline{{ }_{i}(0)}$ for all $t \in[0, T]$ for $i=1,2, \quad \mathbf{y}(0, \cdot)=\mathbf{I}$ (identity) in -

(A5) The convective vector fields $\mathbf{v}_{1}$ and $\mathbf{v}_{2}$, which may occur by the motion of domains, are prescribed so that $\mathbf{v}_{i} \in C^{1}\left(\bar{Q}_{i}\right)^{3}$ for $i=1,2$ and the following properties are satisfied:

$$
\begin{gathered}
\operatorname{div} \mathbf{v}_{i}(t, \cdot)=0 \quad \text { in } \quad i(t) \quad \text { for all } t \in(0, T) \text { for } i=1,2, \\
\mathbf{v}_{1} \cdot \boldsymbol{\nu}^{+}=-\mathbf{v}_{2} \cdot \boldsymbol{\nu}^{-}=v_{\Sigma_{12}} \quad \text { on } \Sigma_{12} \\
\mathbf{v}_{1} \cdot \boldsymbol{\nu}=\mathbf{v}_{2} \cdot \boldsymbol{\nu}=0 \quad \text { on } \Sigma:=(0, T) \times \Gamma,
\end{gathered}
$$

where $v_{\Sigma_{12}}:=v_{\Sigma_{12}}(t, x)$ is the normal speed of $\Gamma_{12}(t)$ at $x \in \Gamma_{12}(t)$. 
Using our abstract theory, we show the existence of a weak solution of (TDP). Let us take the Sobolev space $H^{1}()$ and the Hilbert space $L^{2}()$ as $V$ and $H$, respectively. Then we have

$$
V \stackrel{d}{\hookrightarrow} H \stackrel{d}{\hookrightarrow} V^{*}
$$

where $\left(H^{1}()\right)^{*}=: V^{*}$ is the dual space of $V$. Moreover $\psi^{t}, K, G$ and $f$ are defined as follows:

- $\psi^{t}$ is a proper, lower semicontinuous and convex function on $V^{*}$ defined by

$$
\psi^{t}(u):= \begin{cases}\sum_{i=1,2} \int_{i(t)} \hat{\beta}_{i}(u) d x & \text { if } u \in H, \\ +\infty & \text { if } u \in V^{*} \backslash H,\end{cases}
$$

where $\hat{\beta}_{i}(r):=\int_{0}^{r} \beta_{i}(s) d s$;

- $K$ is a singlevalued operator from $[0, T] \times V$ into $V^{*}$ defined by

$$
\langle K(t, \theta), z\rangle_{V^{*}, V}:=\int k(t, \theta)(\nabla \theta \cdot \nabla z) d x+n_{0} \int_{\Gamma} \theta z d \Gamma \quad \text { for all } z \in V,
$$

where $k(t, \theta):=k(t, x, \theta(x))$ and $k:[0, T] \times \quad \times \mathbf{R} \rightarrow \mathbf{R}$ is defined by

$$
k(t, x, r):=\left\{\begin{array}{lll}
k_{1}(r) & \text { for all } r \in \mathbf{R} & \text { if } x \in{ }_{1}(t), \\
k_{2}(r) & \text { for all } r \in \mathbf{R} & \text { if } x \in \overline{{ }_{2}(t)}
\end{array}\right.
$$

- $G$ is a singlevalued operator from $[0, T] \times H$ into $V^{*}$ defined by

$$
\langle G(t, u), z\rangle_{V^{*}, V}:=-\sum_{i=1,2} \int_{i(t)} u\left(\mathbf{v}_{i}(t) \cdot \nabla z\right) d x \quad \text { for all } z \in V
$$

- $f$ is a function in $L^{2}\left(0, T ; V^{*}\right)$ given by

$$
\langle f(t), z\rangle_{V^{*}, V}:=\int h(t) z d x+\int_{\Gamma} p(t) z d \Gamma \quad \text { for all } z \in V \text { and a.e. } t \in[0, T],
$$

where $h \in L^{2}(Q)$ and $p \in L^{2}(\Sigma)$ are prescribed.

It is easily seen that (A1), (A2) and (A3) are satisfied (cf. T. Fukao, N. Kenmochi and I. Pawtow [10]). Now our problem (TDP) is reformulated in the following weak variational form:

$(5.13)-\int_{Q} u \frac{\partial \eta}{\partial t} d x d t-\int_{Q} u(\mathbf{v} \cdot \nabla \eta) d x d t+\int_{Q} k(\theta)(\nabla \theta \cdot \nabla \eta) d x d t+n_{0} \int_{\Sigma} \theta \eta d \Gamma d t$ 


$$
=\int_{Q} h \eta d x d t+\int_{\Sigma} p \eta d \Gamma d t+\int u_{0} \eta(0) d x \quad \text { for all } \eta \in W
$$

where

$$
\begin{gathered}
k(\theta):=k(t, x, \theta(t, x)), \\
W:=\left\{\eta \in H^{1}(Q) ; \eta(T, \cdot)=0 \text { on } \quad\right\},
\end{gathered}
$$

and

$$
u:=u_{i}, \quad \theta:=\theta_{i}, \quad \mathbf{v}:=\mathbf{v}_{i} \quad \text { on } Q_{i} \quad \text { and } u_{0}:=u_{i 0} \quad \text { on } \quad{ }_{i}(0) \quad \text { for } i=1,2 .
$$

In fact, the variational identity (5.13) is derived from (5.1), (5.2), (5.3) and (5.4) in the following way. Let $\eta$ be any test function in $W$. First, multiply (5.1) and (5.3) by $\eta$ and integrate their resultants over $Q_{1}$ and $Q_{2}$, respectively. Then, with the help of conditions (5.8), (5.10), (5.11), (5.12) and the Green-Stokes' formula we have

$$
\begin{aligned}
\sum_{i=1,2} \int_{Q_{i}}\left(\frac{\partial u_{i}}{\partial t}+\mathbf{v}_{i} \cdot \nabla u_{i}\right) \eta d x d t & \\
= & -\int_{Q_{1}} u_{1}\left(\frac{\partial \eta}{\partial t}+\mathbf{v}_{1} \cdot \nabla \eta\right) d x d t \\
& +\int_{\Sigma_{12}} u_{1} \eta\left(\mathbf{v}_{1} \cdot \nu^{+}\right) d \Gamma_{12}(t) d t-\int_{1(0)} u_{10} \eta(0) d x \\
& -\int_{Q_{2}} u_{2}\left(\frac{\partial \eta}{\partial t}+\mathbf{v}_{2} \cdot \nabla \eta\right) d x d t \\
& +\int_{\Sigma_{12}} u_{2} \eta\left(\mathbf{v}_{2} \cdot \nu^{-}\right) d \Gamma_{12}(t) d t-\int_{2}(0) u_{20} \eta(0) d x \\
= & -\int_{Q} u\left(\frac{\partial \eta}{\partial t}+\mathbf{v} \cdot \nabla \eta\right) d x d t-\int_{u_{0}} \eta(0) d x
\end{aligned}
$$

Next, by (5.7) and (5.9),

$$
\begin{aligned}
- & \sum_{i=1,2} \int_{Q_{i}} \operatorname{div}\left(k_{i}\left(\theta_{i}\right) \nabla \theta_{i}\right) \eta d x d t \\
= & \int_{Q_{1}} k_{1}\left(\theta_{1}\right)\left(\nabla \theta_{1} \cdot \nabla \eta\right) d x d t \\
& -\int_{\Sigma_{1}} k_{1}\left(\theta_{1}\right) \frac{\partial \theta_{1}}{\partial \boldsymbol{\nu}} \eta d \Gamma_{1}(t) d t+\int_{\Sigma_{12}} k_{1}\left(\theta_{1}\right) \frac{\partial \theta_{1}}{\partial \boldsymbol{\nu}^{+}} \eta d \Gamma_{12}(t) d t \\
& +\int_{Q_{2}} k_{2}\left(\theta_{2}\right)\left(\nabla \theta_{2} \cdot \nabla \eta\right) d x d t \\
& -\int_{\Sigma_{2}} k_{2}\left(\theta_{2}\right) \frac{\partial \theta_{2}}{\partial \boldsymbol{\nu}} \eta d \Gamma_{2}(t) d t+\int_{\Sigma_{12}} k_{2}\left(\theta_{2}\right) \frac{\partial \theta_{2}}{\partial \boldsymbol{\nu}^{-}} \eta d \Gamma_{12}(t) d t
\end{aligned}
$$




$$
=\int_{Q} k(\theta)(\nabla \theta \cdot \nabla \eta) d x d t+\int_{\Sigma}\left(n_{0} \theta-p\right) \eta d \Gamma(t) d t .
$$

Here, add the above two equations. Then we get the variational identity (5.13). Moreover by (5.2), (5.4) and the definition of $\psi^{t}$ we see that

$$
\theta(t)=\partial \psi^{t}(u(t)) \text { in } V \text { for a.e. } t \in[0, T],
$$

and (5.13) can be transformed to a doubly nonlinear problem of the form (DN) in the space $V^{*}$. Therefore, applying Theorem 2.1, we conclude that there exist $u \in W^{1,2}\left(0, T ; V^{*}\right) \cap L^{\infty}(0, T ; H)$ and $\theta \in L^{2}(0, T ; V)$ which give a weak solution of (TDP) in the variational sense (5.13).

\section{REFERENCES}

1. T. Arai, On the existence of solution for $\partial \phi\left(u^{\prime}(t)\right)+\partial \psi(u(t)) \ni f(t), J$. Fac. Sci. Univ. Tokyo Sect. IA Math., 26 (1979), 75-96.

2. V. Barbu, Nonlinear Semigroups and Differential Equations in Banach Spaces, Noordhoff, Leyden, 1976.

3. H. Brézis, Équations et inéquations non linéaires dans les espaces vectoriels en dualité, Ann. Inst. Fourier (Grenoble), 18 (1968), 115-175.

4. H. Brézis, Opérateurs Maximaux Monotones et Semi-groupes de contractions dans les espaces de Hilbert, Math. Studies, 5, North-Holland, Amsterdam, 1973.

5. F. E. Browder, Nonlinear equations of evolution, Ann. of Math., 80 (1964), 485-523.

6. F. E. Browder and P. Hess, Nonlinear mappings of monotone type in Banach spaces, J. Funct. Anal., 11 (1971), 251-294.

7. P. Colli, On some doubly nonlinear evolution equations in Banach spaces, Japan J. Indust. Appl. Math., 9 (1992), 181-203.

8. P. Colli and A. Visintin, On a class of doubly nonlinear evolution equations, Comm. Partial differential equations, 15 (1990), 737-756.

9. T. Fukao, Transmission problems for degenerate parabolic equations, pp. 103-112 in Elliptic and Parabolic Problems, World Scientific, New Jersey-London-SingaporeHong Kong, 2002.

10. T. Fukao, N. Kenmochi and I. Pawłow, Transmission problems aris ing in Czochralski process of crystal growth, pp. 228-243 in Mathmatical Aspects of Modeling Structure Formation Phenomena, GAKUTO Internat. Ser. Math. Sci. Appl., Vol. 17, Gakkotosho, Tokyo, 2002.

11. T. Fukao, N. Kenmochi and I. Pawłow, Mathematical modeling for Czochralski process of crystal growth, Preprint. Istituto di Analisi Numerica del Consiglio Nazionale delle Ricerche, Pavia, 1279 (2002), 1-16. 
12. T. Fukao, N. Kenmochi and I. Pawłow, Transmission-Stefan problems arising in Czochralski process of crystal growth, to appear in Internat. Ser. Numer. Math., Birkhäuser.

13. T. Fukao, N. Kenmochi and I. Pawłow, Stefan problems in non-cylindrical domains arising in Czochralski process of crystal growth, to appear in Control Cybernet., 32 (2003).

14. N. Kenmochi, Existence theorems for certain nonlinear equations, Hiroshima Math. J., 1 (1971), 435-443.

15. N. Kenmochi, Nonlinear operators of monotone type in reflexive Banach spaces and nonlinear perturbations, Hiros hima Math. J., 4 (1974), 229-263.

16. N. Kenmochi, Solvability of nonlinear evolution equations with time-dependent constraints and applications, Bull. Fac. Edu, Chiba Univ., 30 (1981), 1-87.

17. N. Kenmochi and I. Pawłow, A class of nonlinear elliptic-parabolic equations with time-dependent constraints, Nonlinear Anal., 10 (1986), 1181-1202.

18. N. Kenmochi and I. Pawłow, Parabolic-eliptic free boundary problems with timedependent obstacles, Japan J. Appl. Math., 5 (1988), 87-121.

19. E. Maitre and P. Witomski, A pseudo-monotonicity adapted to doubly nonlinear elliptic-parabolic equations, Nonlinear Anal., 50 (2002), 223-250.

20. M. Ôtani, Nonmonotone perturbations for nonlinear parabolic equations associated with subdifferential operators, Cauchy problems, J. Differential Equations, 46 (1982), 268-299.

21. T. Senba, On some nonlinear evolution equation, Funk. Ekvac., 29 (1986), 243-257.

22. Y. Yamada, On evolution equations generated by subdifferential operators, J. Fac. Sci. Univ. Tokyo Sect. IA Math., 23 (1976), 491-515.

\author{
Masayasu Aso \\ Graduate School of Science and Technology, Chiba University \\ 1-33 Yayoi-cho, Inage-ku, Chiba, 263-8522 Japan \\ E-mail: masayasu.aso@graduate.chiba-u.jp \\ Takesi Fukao \\ Department of Mathematics, Faculty of Education, \\ Chiba University, \\ 1-33 Yayoi-cho, Inage-ku, Chiba, 263-8522, Japan \\ E-mail: fukao@faculty.chiba-u.jp \\ Nobuyuki Kenmochi \\ Department of Mathematics, Faculty of Education, \\ Chiba University, \\ 1-33 Yayoi-cho, Inage-ku, Chiba, 263-8522, Japan \\ E-mail: kenmochi@faculty.chiba-u.jp
}

